\title{
CROSSOVER FROM BCS TO LOCAL PAIR SUPERCONDUCTIVITY IN QUASI-2D SYSTEMS
}

\author{
E.V.Gorbar, V.M.LOKTEV S.G.SHARAPOV \\ N.N.Bogolyubov Institute for Theoretical Physics \\ Ukrainian National Academy of Sciences \\ $14^{b}$ Metrologichna St., 252143 Kiev-143, Ukraine
}

Received September 12, 1995

\begin{abstract}
The finite temperature crossover from cooperative Cooper pairing to independent bound state (composite bosons) formation and condensation in quasi-2D systems is studied using the method of effective potential. It is shown that at low density of carriers the critical superconducting temperature is equal to that of Bose-condensation for ideal quasi-2D Bose-gas with heavy dynamical mass, meanwhile at high densities the BCS result remains valid.
\end{abstract}

1. The problem of crossover from the BCS theory with cooperative Cooper pairing to formation and condensation of composite bosons [1,2] (see also the review [3]) remains of interest for a long time. After the discovery of high-temperature superconductivity (HTSC) much attention was paid to this problem [4-6]. For example, it was shown in [5], using the BetheSalpiter equation, that real bound states in r-space are formed in a 2Dsuperconductor as the chemical potential $\mu$ takes negative values.

For pure 2D superconductors, the transition from the regime of local pairs to the Cooper pairing was studied in Refs. [4,5] in the mean field (MF) approximation. However, it is well known that this approximation for a 2D problem is not valid for instance because of the following reasons:

i) firstly, by virtue of the Coleman-Mermin-Wagner-Hohenberg theorem [7] a "super-behaviour" is not possible for pure 2D systems [8] due to the fluctuations of order parameter (especially of its phase) [9]. Of course, any real system is not $2 \mathrm{D}$ but it is at least a quasi- $2 \mathrm{D}$ one that, to be more specific, was neglected in Refs. [4,5], where the case of $T=0$ was considered.

i2) secondly, even for 3D systems the MF approximation in the case of local pairs superconductivity proves to be wrong [6]: in fact, instead of the true critical temperature $T_{c}$ it results in the value of the temperature for the local pair formation.

Taking into account the Gauss fluctuations, and study of the problem of crossover was carried out for a 3D system in Ref. [6]. However, it is necessary to note that the HTSCs, study of such a crossover for which is the most actual now, are not ordinary 3D systems but quasi-2D ones because of the essential (up to five orders) anisotropy of the conductivity. Therefore, from our point of view, the generalization of results of Ref. [6] for the case of quasi-2D systems would be of significant interest.

(C) E.V.Gorbar, V.M.Loktev S.G.Sharapov, 1996

ISSN 0452-9910. Condensed Matter Physics 1996 No 7 (53-59) 
In this paper we research a model of a quasi-2D superconductor taking into account the fluctuations of the order parameter in the random phase approximation using the Coleman-Weinberg method of effective potential. We show that in the case of high carrier density $n$ when $\mu \rightarrow \epsilon_{F}\left(\epsilon_{F}\right.$ is the Fermi energy) the critical temperature $T_{c}$ is determined by the usual BCS formula and increases with rise in of attraction between fermions. In the opposite limit - the case of local pairs $-T_{c}$ is determined by the formula for the Bose-condensation temperature for quasi-2D Bose-gas [10]. It is important that in the latter case $T_{c}$ decreases at rise of such an attraction. It is likely that the intermediate region which lies between limiting cases abovementioned is more relevant to HTSCs for which the value of $T_{c}$ is probably close to its maximum one.

2. The simplest model Hamiltonian for carriers (electrons or holes) in the system reads

$$
\begin{aligned}
H= & -\psi_{\sigma}^{\dagger}(x)\left[\frac{\nabla_{\perp}^{2}}{2 m_{\perp}}-\frac{1}{m_{z} d^{2}} \cos \left(\imath \nabla_{z} d\right)+\mu\right] \psi_{\sigma}(x)- \\
& -V \psi_{\uparrow}^{\dagger}(x) \psi_{\downarrow}^{\dagger}(x) \psi_{\downarrow}(x) \psi_{\uparrow}(x),
\end{aligned}
$$

where $x \equiv t, \mathbf{r}_{\perp}, r_{z}$ (with $\mathbf{r}_{\perp}$ being a $2 \mathrm{D}$ vector); $\psi_{\sigma}(x)$ is a fermion field in the Shrödinger representation, $\sigma$ is the spin variable; $m_{\perp}$ is an effective mass of carriers in planes (for example, $\mathrm{CuO}_{2}$ ones); $m_{z}$ is an effective mass in $z$ direction; $d$ is the interlayer distance; $V$ is an effective local attraction inside planes; $\mu$ and $n$ are determined above; one also assumes that $\hbar=k_{B}=1$.

It is significant that the large anisotropy of conductivity cannot be identified with the similar anisotropy of the effective masses. In particular, HTSCs with large anisotropy in z-direction do not display the metal behaviour, which shows that interplane motion of particles is incoherent. But, as will be seen, to justify approximations used below the not very large ratio $m_{z} / m_{\perp} \geq 10^{2}$ is already sufficient. Namely such a value is attained, for instance, in HTSC 1-2-3.

Lagrangian density connected with (1) has the following standard form:

$$
L=\imath \psi_{\sigma}^{\dagger} \partial_{t} \psi_{\sigma}-H, \quad \psi_{\sigma} \equiv \psi_{\sigma}(x) .
$$

Introducing an auxiliary Hubbard-Stratonovich field $\Phi \equiv V \psi_{\uparrow}^{\dagger} \psi_{\downarrow}^{\dagger}$ and integrating over the fermions, one obtains the following effective action

$$
S_{e f f}\left(\Phi, \Phi^{*}\right)=-\imath \operatorname{Tr} \operatorname{Ln} G^{-1}-\frac{1}{V} \int d t d \mathbf{r}|\Phi|^{2}
$$

with

$$
\begin{aligned}
G^{-1}=\imath \frac{\partial}{\partial t} I & +\left(\frac{\nabla_{\perp}^{2}}{2 m_{\perp}}-\frac{1}{m_{z} d^{2}} \cos \left(\imath \nabla_{z} d\right)+\mu\right) \tau_{3}+ \\
& +\frac{\tau_{1}+\imath \tau_{2}}{2} \Phi+\frac{\tau_{1}-\imath \tau_{2}}{2} \Phi^{*}
\end{aligned}
$$

where $I$ is the unity matrix, $\tau_{j}$ are the Pauli matrices. The effective potential $U_{M F}\left(\Phi, \Phi^{*}\right)$ which describes the system with homogeneous order parameter in the MF approximation is determined from (2) by the following relation:

$$
S_{e f f}\left(\Phi, \Phi^{*}\right)=\int d t d \mathbf{r}\left[T_{k i n}\left(\Phi, \Phi^{*}, \nabla \Phi, \nabla \Phi^{*}\right)-U_{M F}\left(\Phi, \Phi^{*}\right)\right]
$$


where $T_{k i n}$ is a power series in derivatives ${ }^{1}$.

Applying the usual finite-temperature formalism [11], the equation $\partial U_{M F} /\left.\partial \Phi\right|_{\Phi=\Phi^{*}=0}=0$ transforms to the standard gap equation

$$
\frac{1}{V}=\int \frac{d \mathbf{k}}{(2 \pi)^{3}} \frac{1}{2 \xi(\mathbf{k})} \tanh \frac{\xi(\mathbf{k})}{2 T_{c}^{M F}}
$$

where $\xi(\mathbf{k})=\varepsilon(\mathbf{k})-\mu$ with $\varepsilon(\mathbf{k})=\mathbf{k}_{\perp}^{2} / 2 m_{\perp}+1 /\left(m_{z} d^{2}\right) \cos \left(k_{z} d\right)$ (reason by which we use $T_{c}^{M F}$ rather than $T_{c}$ will be clarified in what follows). We would like to emphasize that under temperatures of interest, the band width in the $z$-direction is $m_{z}^{-1} d^{-2} \ll T_{c}^{M F}$. As for the last inequality, it is easy to see, that at $m_{z} \approx 10^{2} m_{e}$ and $d=10 \dot{A}$ the value $\hbar^{2} /\left(m_{z} d^{2} k_{B}\right) \sim 10 K$ is really lesser then the usual critical temperatures in HTSC compounds.

To study equation (4) in the case of the local pairs superconductivity the cutoff of BCS type can not be applied. In order to eliminate the divergences in (4) it is convenient to introduce (see Miyake in [1] and [4,5]) the energy of a two-particle bound state $\varepsilon_{b}=-2 W \exp \left(-4 \pi d / m_{\perp} V\right)$, where $W=$ $\mathbf{k}_{\perp \max }^{2} / 2 m_{\perp}$ is the band width in the plane ${ }^{2}$. Then, expressing the lefthand side of equation (4) through $\left|\varepsilon_{b}\right|$ and performing the limit $W \rightarrow \infty$, one can easy regularize the gap equation. The physical meaning of this limiting procedure is based on enough natural assumption that $W \gg \epsilon_{F}$.

On the contrary to the usual BCS approach in which it is always assumed that $\mu=\epsilon_{F}$, here the value of chemical potential should be consistently defined from the equation $\partial U_{M F} /\left.\partial \mu\right|_{\Phi=\Phi^{*}=0}=-n$, which leads to another one, or the number equation:

$$
n_{f}\left(\mu, T_{c}^{M F}\right) \equiv \int \frac{d \mathbf{k}}{(2 \pi)^{3}}\left[1-\tanh \frac{\xi(\mathbf{k})}{2 T_{c}^{M F}}\right]=n
$$

In such a case one needs to solve simultaneously the system of equations (4), (5) with two unknown variables $-T_{c}^{M F}$ and $\mu \equiv \mu\left(T_{c}^{M F}\right)$, respectively (in general case of arbitrary $T$ chemical potential $\left.\mu \equiv \mu(T) \neq \epsilon_{F}\right)$.

At high concentrations $n$, so that $\mu \gg T_{c}^{M F}$, equality $\mu \simeq \epsilon_{F}$ indeed is the solution of equation (5), where $\epsilon_{F}=\pi n d / m_{\perp}$ is the Fermi energy of free $2 \mathrm{D}$ fermions with $\varepsilon(\mathbf{k}) \sim k^{2}$. Taking into account the regularization procedure, it follows from (4) that $T_{c}^{M F} \simeq \gamma \pi^{-1} \sqrt{2\left|\varepsilon_{b}\right| \epsilon_{F}}$ with $\gamma \simeq 1.781$. This is just well known BCS result for a $2 \mathrm{D}$ metal $[4,5]$.

In the opposite case of small concentrations, such as $-\mu \gg T_{c}^{M F}$, the roles of the gap (4) and number (5) equations are reversed: the equation (4) determines $\mu$, while the equation (5) determines $T_{c}^{M F}$. Then, from the gap equation, one can obtain that $\mu=-\left|\varepsilon_{b}\right| / 2-m_{z}^{-2} d^{-4}\left|\varepsilon_{b}\right|^{-1} / 2$. The latter except for the second term corresponds to the result of Ref. [6]. It is directly connected with the quasi-2D character of the model and (despite this term is essentially less than the first one) is very important in the case when the fluctuations are taken into account. At $\left|\varepsilon_{b}\right| \gg \epsilon_{F}$ equation (4) transforms into the transcendent one: $\left|\varepsilon_{b}\right| / 2 T_{c}^{M F}=\ln \left(T_{c}^{M F} / \epsilon_{F}\right)$. Hereafter, we directly obtain the above result: for a finite energy $\epsilon_{F}, T_{c}^{M F}$ strongly grows as $V$

\footnotetext{
[5].

${ }^{1}$ For the case of a $2 \mathrm{D}$ system at $T=0$, the first terms of this series is found in Ref.

${ }^{2}$ Note that in the region of parameters considered these bound states form in what follows without threshold.
} 
increases. Thus, for the case of a small carrier density the temperature $T_{c}^{M F}$ is not connected with the critical one $T_{c}$ and in fact it corresponds to the temperature of composite boson dissociation [6].

3. In order to allow for the effects of composite bosons formed in the system, we apply the Coleman-Weinberg method of the effective action [12]. In this method the effective potential $U\left(\Phi, \Phi^{*}\right)=U_{M F}\left(\Phi, \Phi^{*}\right)+U^{(1)}\left(\Phi, \Phi^{*}\right)$, where $U_{M F}$ is the "tree-potential" (i.e., it is obtained in the MF approximation) and $U^{(1)}$ is one-loop correction to it. At arbitrary $T$ 's

$$
\begin{aligned}
& U^{(1)}\left(\Phi, \Phi^{*}\right)= \\
& \frac{-\imath}{2} \int \frac{d^{4} q}{(2 \pi)^{4}} \ln \left[\left(\frac{1}{V}-\frac{\imath}{(2 \pi)^{4}} \int d^{4} p \operatorname{tr}\left[G(p) \tau_{+} G(p+q) \tau_{-}\right]\right)^{2}\right. \\
& \left.-\left(\frac{\imath}{(2 \pi)^{4}} \int d^{4} p \operatorname{tr}\left[G(p) \tau_{+} G(p+q) \tau_{+}\right]\right)^{2}\right], \\
& p \equiv p_{0}, \mathbf{p}, \quad q \equiv q_{0}, \mathbf{q},
\end{aligned}
$$

where $p_{0}=\imath \pi(2 j+1) T, q_{0}=\imath 2 \pi l T(j, l$ - integers $), \tau_{ \pm}=\left(\tau_{1} \pm \imath \tau_{2}\right) / 2$, type- $G(p)$ and $G(p+q)$ are Green functions from (3) in the frequency and momentum representation. Expression (6) can be useful to study how the energy gap $|\Phi|$ depends on the temperature. In what follows, we restrict ourselves by the case of critical line $\Phi=\Phi^{\star}=0$ only and investigate the effect of the fluctuations on $T_{c}$. In so doing case one obtains

$$
U^{(1)}\left(\Phi=\Phi^{*}=0\right)=-\imath \int \frac{d^{4} q}{(2 \pi)^{4}} \ln D^{-1}\left\{q_{0}, \mathbf{q}\right\}
$$

where

$$
\begin{aligned}
D^{-1}\left\{q_{0}, \mathbf{q}\right\}=\frac{1}{V}-\frac{1}{2} \int & \frac{d \mathbf{p}}{(2 \pi)^{3}} \frac{1}{\xi(\mathbf{p}-\mathbf{q} / 2)+\xi(\mathbf{p}+\mathbf{q} / 2)-q_{0}} \times \\
& {\left[\tanh \frac{\xi(\mathbf{p}-\mathbf{q} / 2)}{2 T_{c}}+\tanh \frac{\xi(\mathbf{p}+\mathbf{q} / 2)}{2 T_{c}}\right] . }
\end{aligned}
$$

Further, one has to remove the divergences from (7) applying the same procedure of regularization as in case of the gap equation (4).

According to [2], we introduce the phase $\delta(\omega, \mathbf{q}) \equiv-\arg D^{-1}\{\omega+\imath 0, \mathbf{q}\}$ and retard the contribution of fluctuations through the number equation (5)

$$
\begin{aligned}
n_{f}\left(\mu, T_{c}\right) & +2 n_{B}\left(\mu, T_{c}\right)=n \\
n_{B}\left(\mu, T_{c}\right) & \equiv \frac{1}{2} \int \frac{d \mathbf{q}}{(2 \pi)^{3}} \int_{-\infty}^{\infty} \frac{d \omega}{\pi} n_{B}(\omega) \frac{\partial \delta(\omega, \mathbf{q})}{\partial \mu}
\end{aligned}
$$

where $n_{B}(\omega) \equiv[\exp (\omega / T)-1]^{-1}$ is the Bose distribution function. One can see from (8) that the system of fermions is divided into two coexisting and dynamically bounded systems: fermi-particles, or unbounded fermions, and local pairs or bosons. Therewith the corresponding concentrations of fermi- and bose-particles depend on $T$ and only mean values are known. 
4. Consider the influence of boson contribution $n_{B}\left(\mu, T_{c}\right)$ on the behaviour of the system as the carrier density changes. Then we should consistently solve the system of equations (4) and (8). At a fairly high carrier density such a contribution, $n_{B}\left(\mu, T_{c}\right)$ in (8), is small, and we obtain that $T_{c} \simeq T_{c}^{M F}$. It is worth to note the following: in more consistent scheme one should take into consideration the correction $\partial U^{(1)} / \partial \Phi$ in the gap equation (4), and consequently the fluctuations would modify it. However, because of the conditions $\mu=\epsilon_{F} \gg T_{c}$ and $m_{z} d^{2} T_{c} \gg 1$, one can easily see that this correction changes $T_{c}$ rather weakly [13] (see also [14]). It follows, from the BCS expression obtained for $T_{c}^{M F}$ in this limit (the $T_{c}^{M F}$ coincides with the expression obtained in Ref. [5] for a pure 2D superconductor due to the band narrowness in the $k_{z}$-direction) that $T_{c}$ increases when the couple constant $V$ rises up.

At small concentrations, such that $|\mu| / T_{c} \gg 1$, the contribution of bosons, contrary to the previous case, is dominant and (remind that in this limiting case $\mu$ is negative), so one has from (8) that

$$
n_{B}\left(\mu, T_{c}\right) \equiv \int \frac{d \mathbf{q}}{(2 \pi)^{3}} n_{B}\left(\frac{\mathbf{q}_{\perp}^{2}}{4 m_{\perp}}+\frac{1}{2\left|\varepsilon_{b}\right|\left(m_{z} d^{2}\right)^{2}}\left[1-\cos \left(q_{z} d\right)\right]\right) \simeq \frac{n}{2},
$$

i.e. all fermions are bounded between themselves forming composite bosons. It is easy to see that the boson effective mass for its motion in the plane retains the value $2 m_{\perp}$. As to the motion between the planes, the effective boson mass increases considerably: $2\left|\varepsilon_{b}\right| m_{z}^{2} d^{2}\left(\gg m_{z}\right)$. It is worth to stress that this increasing has the dynamical character what is simply testified by the presence of $\left|\varepsilon_{b}\right|$. Physically, it is ensured by the one-particle character (see equation (1)) of the interplane tunnelling. Consequently, it is connected for a pair, with its virtual breakup, for which the energy loss is of the order $\left|\varepsilon_{b}\right|$.

Now, using the formula for the Bose condensation temperature of an ideal quasi-2D Bose-gas [10], it is easy to write down an equation for determining $T_{c}$, which in the case under consideration takes the form

$$
T_{c} \simeq \frac{\pi n d}{2 m_{\perp} \ln \left(2 T_{c}\left|\varepsilon_{b}\right| m_{z}^{2} d^{4}\right)}=\frac{\epsilon_{F}}{2 \ln \left(2 T_{c}\left|\varepsilon_{b}\right| m_{z}^{2} d^{4}\right)} .
$$

The last equation describes the characteristic properties of a quasi-2D superconductor with small carrier density: $\imath$ ) firstly, as the critical temperature $T_{c} \sim \epsilon_{F}$, or (see above) $T_{c} \sim n$, just as it should be in $2 \mathrm{D}$ case (remind that in a $3 \mathrm{D}$ one $T_{c} \sim n^{2 / 3}[6]$ ), and in MF approximation $2 \mathrm{D} T_{c}^{M F} \sim \sqrt{n} \gg T_{c}$ [5]; $\imath 2)$ secondly, on the contrary to the case of $3 \mathrm{D}$ superconductor where $T_{c}$ does not depend on $V$ at all [6], in a quasi-2D system $T_{c}$ is irrespective of $V$, namely: $T_{c}$ falls off with the rise in $V$. As it was stated above, the reason for this is the dynamical increasing of the composite boson mass along the third direction. Thus, the growth of $\left|\varepsilon_{b}\right|$ (or of $V$, what is the same) "makes" the system more and more two-dimensional one even for the simplest case of quasi-2D metal with a local four-fermion interaction.

It is interesting to note that decreasing $T_{c}$ can also take place in the case when the local pairs are bipolarons [15]; then, the increasing of coupling with phonons, which makes the pairs more massive, also leads to $T_{c}$ decreasing, (rather than increasing) as the electron-phonon coupling grows.

5. The results obtained show that in order to obtain the maximum $T_{c}$ in a quasi-2D system with local composite boson (independently of its type) the 
attraction should have an optimal value. It is possible that it gets into the region of crossover when the system is in an intermediate regime $\epsilon_{F} \approx\left|\varepsilon_{b}\right|$. However, this region of parameters needs a special study because of both presence of strongly developed fluctuations and possible distinction of properties of such a Fermi-liquid from the Fermi-liquid of Landau type. Note, in conclusion, that the fluctuations might also enhance non-monotonic character of the $T_{c}$ behaviour versus $n$ obtained for many-layers HTSC models [16].

We are grateful to Profs. V.P. Gusynin, E.A. Pashitskii, I.I. Ukrainskii and Dr. I.A. Shovkovy for numerous discussion and fruitful remarks. The present research was made possible in part by Grant No K5O100.

\section{References}

[1] Eagles D.M. Possible pairing without Superconductivity at Low Carrier Concentrations in Bulk and Thin-Film. // Phys. Rev., 1969, vol. 186, No 2, p. 456-463;

Leggett A.J. in: Modern Trends in Theory of Condensed Matter, edited by A. Pekalski and R. Przystawa. Berlin, Springer-Verlag, 1980, p. 13 - 41;

Miyake K. Fermi Liquid Theory of Dilute Submonolayer ${ }^{3} \mathrm{He}$ on Thin ${ }^{4} \mathrm{He}$ II Film. // Progr. Theor. Phys., 1983, vol. 69, No 6, p. 1794-1797.

[2] Nozieres P., Schmitt-Rink S. Bose-condensation in an attractive Fermion gas: from weak to strong coupling Superconductivity. // J. Low Temp. Phys., 1985, vol. 59, No 3/4, p. 195-211.

[3] Mičnas R., Ranniger J., Robaszkievicz S. Superconductivity in Narrow-Band Systems with Local Nonretarded Attractive Interactions. // Rev. Mod. Phys., 1990, vol. 62, No 1, p. 113 - 171.

[4] Randeria M., Duan J.-M., Shieh L. Bound States Cooper Pairing and Bose Condensation in Two Dimensions. // Phys. Rev. Lett., 1989, vol. 62, No 9, p. 981-984; Superconductivity in a two-dimensional Fermi gas: Evolution from Cooper pairing to Bose condensation. // Phys. Rev., 1990, vol. B41, No 1, p. 327-343.

[5] Gorbar E.V., Gusynin V.P., Loktev V.M. Pairing and superconductivity properties of 2D Fermi system with attraction. // Low Temp. Phys., 1993, vol. 19, No 11, p. 832-838.

[6] Sá de Melo C.A.R., Randeria M., Engelbrecht J.R. Crossover from BCS to Bose superconductivity: Transition Temperature and Time-Dependent Ginzburg-Landau Theory. // Phys. Rev. Lett., 1993, vol. 71, No 19, p. 32023205.

[7] Coleman S. There are no Goldstone Bosons in Two Dimensions. // Comm. Math. Phys., 1973, vol. 31, No 4, p. 259-264;

Mermin N.D., Wagner H. Absence of Ferromagnetism or Antiferromagnetism in One- or Two-Dimensional Isotropic Heisenberg Model. // Phys. Rev. Lett., 1966, vol. 17 , No 22, p. 1133-1136;

Hohenberg P.C. Existence of Long-Range Order in One and Two Dimensions. // Phys. Rev., 1967, vol. 158, No 2, p. 383-386.

[8] In the last time, purely 2D models of superconductivity are also considered. These models do not require an homogeneous order parameter and are based on Berezinskii-Kosterlitz-Thouless phase transition: Berezinskii V.L. Destroying of Long-Range Order in One- and Two-Dimensional Systems with Continuous Group Symmetry. I. Classical Systems. // Zh. Eksp. Teor. Fiz., 1970, vol. 59, No 3, p. 907-920;

Kosterlitz J., Thouless D. Ordering, Metastability and Phase Transitions in Two-Dimensional Systems. // J. Phys., 1973, vol. C6, No 7, p. 1181-1203..

[9] Rice T.M. Superconductivity in One and Two Dimensions. // Phys. Rev., 1965 , vol. 140 , No 6 A, p. $1889-1891$.

[10] Wen X.-G., Kan R. Charged Boson Condensation in High- $T_{c}$ Superconductors. // Phys. Rev., 1988, vol. B37, No 1, p. 595-598. 
[11] Schrieffer R. Theory of Superconductivity. New-York, Benjamin, 1964.

[12] Miransky V.A. Dynamical Symmetry Breaking in Quantum Field Theory. Singapore, World Scientific Co., 1993.

[13] Dzyaloshinskii I.E., Kats E.I. On Theory of Superconductivity in Quasi-OneDimensional Structures. // Zh. Eksp. Teor. Fiz., 1968, vol. 55, No 12, p. 2373-2375.

[14] Vonsovsky S.V., Izymov Yu.A., Kurmaev E.Z. Superconductivity of Transition Metals its Alloys and Compounds. Moscow, Nauka, 1977.

[15] Alexandrov A.S., Krebs A.B. Polarons in High-temperature Superconductors. // Usp. Fiz. Nauk, 1992, vol. 162, No 5, p. 1-85.

[16] Gorbar E.V., Loktev V.M., Sharapov S.G. Electronic Spectrum and Critical Temperature of the HTSC with a few cuprate layers in a Cell. // Low Temp. Phys., 1995, vol. 21, No 4.

\title{
КРОСОВЕР ВІД БКШ ДО ЛОКАЛЬНОЇ \\ ПАРНОЇ НАДПРОВІДНОСТІ У КВАЗІДВОВИМІРНИХ СИСТЕМАХ
}

\author{
Е.В.Горбар, В.М.Локтєв, С.Г.Шарапов
}

\begin{abstract}
Методом ефективного потенціалу досліджується кросовер при скінченній температурі від кооперативного куперівського спарювання до утворення незалежного граничного стану (композитні бозони) і конденсації в квазідвовимірних системах. Показано, що при низькій густині носіїв, критична температура надпровідності дорівнює температурі бозе-конденсації ідеального квазідвовимірного бозе-газу з високою масою, в той час як при високих густинах результат БКШ залишається справедливим.
\end{abstract}


